\title{
Experience-Based Trust in E-Commerce
}

\author{
Zhaohao Sun ${ }^{1}$, Sukui Lu ${ }^{2}$ Jun $\mathrm{Han}^{3}$, Gavin Finnie ${ }^{4}$ \\ 1 Dept. of Computer Science, College of Mathematics and Information \\ Science, Hebei Normal University, Shijiazhuang, China, 050016, \\ zhsun@ieee.org \\ 2 College of Mathematics and Computer, Hebei University, Baoding, \\ China, 071002, lsk@mail.hbu.edu.cn \\ 3 School of Computer Science, Beihang University, Beijing 100083, China, \\ jun_han@buaa.edu.cn \\ 4 School of Information Technology, Bond University, Gold Coast Qid \\ 4229 Australia \\ gfinnie@staff.bond.edu.au
}

\begin{abstract}
Trust is significant for e-commerce and has received increasing attention in e-commerce, multiagent systems, and artificial intelligence (AI). However, little aftention has been given to the theoretical foundation and intelligent techniques for trust in e-commerce from either a logical or intelligent systems viewpoint. This paper will fill this gap by examining knowledge-based trust, inference-based trust, case-based trust and experience-based trust in e-commerce and their interrelationships from an intelligent systems viewpoint. The proposed approach will facilitate research and development of trust, multiagent systems, e-commerce and e-services.
\end{abstract}

\section{Introduction}

Trust is significant for healthy development of e-commerce. Castelfranchi and Tan [2] assert that e-commerce can be successful only if the general public trusts in the virtual environment, because lack of trust in security is one of the main reasons for econsumers and companies not to engage in e-commerce. Therefore, trust has received increasing attention in e-commerce and information technology (IT). For example, Salam et al [10] examine trust in e-commerce and note that "many customers may still not trust vendors when shopping online". Pavlou [9] integrates

Please use the following format when citing this chapter:

Sun, Z., Lu, S., Han, J., Finnie, G., 2007, in IFIP International Federation for Information Processing, Volume 251, Integration and Innovation Orient to F-Socicty Volumel, Wang, W. (Eds), (Boston: Springer), pp. 643-651. 
trust with the technology acceptance model to explore the customer acceptance of ecommerce. Xiu and Liu [22] propose a formal definition of trust and discuss the properties of trust relation. Wingreen and Baglione [21] study the customer's trust in vendors (knowledge-based trust) from a business viewpoint. Olsson [8] examines trust in e-commerce and asserts that "if experience-based trust (EBT) can be evaluated automatically, then it would provide a foundation for decision support tool for e-commerce customers". Xiong and Liu [23] propose a formal reputation-based trust model by combining amount of satisfaction, number of interaction and balance factor of trust in a peer-to-peer e-communities. However, the majority of works is on trust in online purchase settings, while there is relatively less research on theoretical foundations of trust in e-commerce.

Multiagent systems (MAS) has been successfully applied in many fields such as e-commerce [14]. On the other hand, trust has also drawn some intention in MAS. Tweedale and Cutler discuss trust in MAS by proposing a trust negotiation and communication model for MAS architecture [18]. Chen et al [3] propose a fuzzy trust model for MAS taking into account direct trust, recommendation trust and selfrecommendation trust. Schmidt et al [11] apply a fuzzy trust model to an ecommerce platform. This paper will examine trust in multiagent e-commerce systems (MECS) by examining experience-based trust and case-based trust in ecommerce and their interrelationships from an intelligent systems viewpoint. It also proposes knowledge-based and inference-based trust in e-commerce.

The rest of this paper is organized as follows: Section 2 examines trust in ecommerce from two different perspectives. Section 3 proposes an intelligent model of trust in e-commerce. Section 4 examines experience-based trust. Section 5 introduces a measure model for trust in e-commerce. Section 6 proposes a case-based model for trust in e-commerce and Section 7 concludes the paper with some concluding remarks and future work.

\section{Two Perspectives to Trust in E-Commerce}

Trust is an important ingredient in e-commerce, because the Internet is a source of trust and mistrust [19]. Therefore trust is essential for the success of e-commerce.

There are three main parties involved in e-transactions in e-commerce: the buyer, seller and intermediary [20]. In this study, buyers are either e-buyers or online customers or intelligent buyer agents [14]. Sellers are either Web merchants or Internet stores [12] or intelligent seller agents [14] or Web vendors [5], or online shops websites. Intermediaries are either e-agents or e-facilitors [20] or e-brokers or intelligent agents for any intermediary [15]. This consideration motivates the model of trust in e-commerce from a people-centered viewpoint as shown in Fig. 1.

The interrelationships between buyer trust, intermediary trust and seller trust have received substantial attention in e-commerce. For example, Verhagen, Meets \& Tan [20] explore intermediary trust and seller trust. Intermediary trust refers to "the trustworthiness of the intermediary operating the system. It reflects perceptions of security during transaction to the presence of guarantees, regulations, safety nets or 
other structures that are introduced by these institutions" [20]. Intermediary trust is related to buyer trust and seller trust.

Seller trust refers to the "perceptions of trust in the counterpart of transaction" [20]. More specifically, it refers to the subjective belief with which consumers assess that sellers will perform potential transactions according to their confident expectations, irrespective of their ability to fully monitor them [20]. In e-commerce, the object of seller trust is the party selling the products. Trust in e-sellers is important in consumer-oriented e-commerce adoption decisions [12].

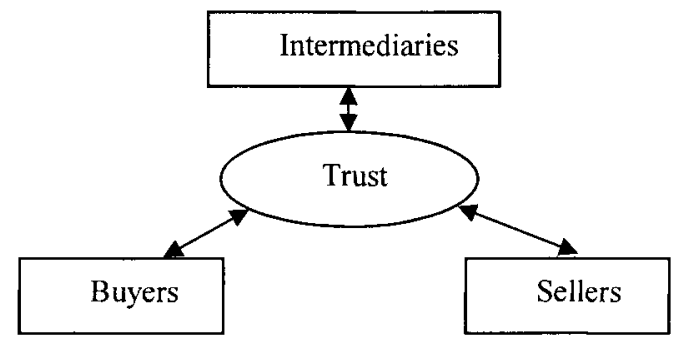

Fig. 1 A people-based model for trust in e-commerce [15]

In this model, buyer $B$, seller $S$ and intermediary $I$ are working together in ecommerce in order to complete an e-business transaction. However, all of them face trust issue in either e-commerce or MECS. Sometimes, they trust each other, sometimes they distrust one another. If they trust each other, it is easy for them to complete the e-business transaction satisfactorily. If they distrust each other, one of them might obtain the interest or benefit from the e-business transaction at one time.

From a system viewpoint, trust exists in the Web client, data transport, Web server, and operating system respectively. At the same time, trust constitutes a chain, which can be called a trust chain. The trust chain links Web client trust, data transport trust, Web server trust, and operating system trust as shown in Fig.2.

Trust can be propagated along the trust chain from Web client trust through data transport trust and Web server trust to operating system trust [16]. This trust propagation affects the trust of customer in the Web client, data transport, Web server, and operating system sequentially in general, in e-commerce in particular. At the same time, this trust propagation also promotes the improvement of the Web browser, data transport, Web server, and operating system. Therefore, the propagation of trust plays a vital role in e-commerce not only from the seller and buyer viewpoint, but also from a viewpoint of researcher or technology of ecommerce.

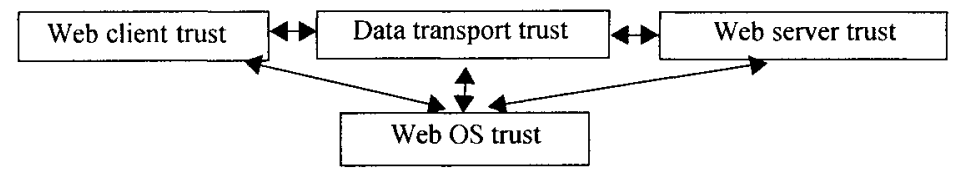

Fig. 2. A trust chain in e-commerce [15] 


\section{An Intelligent Model of Trust in E-Commerce}

We assume that $P$ is an agent and $Q$ is another agent. $P$ has a knowledge set $K_{P}$, which can be considered as the knowledge base in a knowledge based agent, reasoning methods set $R_{P}$, which can be considered the problem-solving methods. $Q$ has knowledge base $K_{Q}$ and reasoning set $R_{Q}$. Therefore, from a knowledge based systems viewpoint, the behavior of $P$ and $Q$ will be decided by $\left(K_{P}, R_{P}\right)$ and $\left(K_{Q}, R_{Q}\right)$ under the same environment.

It should be noted that $K_{P}$ can also include one's experience, data, and information. $R_{P}$ also consists of non-traditional reasoning methods.

Now we consider trust between $P$ and $Q$.

As is well known, trust is a binary relation [22]. For example, agent $P$ trusts agent $Q$. However, few have gone into this binary relation.

In the most general case, one of the essentially necessary conditions for "agent $P$ trusts agent $Q$ " is that agent $Q$ has more knowledge and reasoning methods or problem solving methods than agent $P$, because this is the important premise of agent $P$ placing confidence in agent $Q$ [1]. In other words, a necessary condition for "agent $P$ trusts agent $Q$ " is that at the time $t$, agent $P$ and agent $Q$ satisfy:

$K_{P} \subseteq K_{Q}$ and $R_{P} \subseteq R_{Q}$

Based on (1), we can see that trust as a binary relation satisfying:

1. Reflectivity. Agent $P$ trusts agent $P$ itself.

2. Anti-symmetry. If agent $P$ trusts agent $Q$, and agent $Q$ trusts agent $P$, then $P=Q$. This is usually inconsistent with reality, because in e-commerce, agent $P$ and agent $Q$ can trust each other for an e-transaction. However, there are really many cases in e-commerce, in which, agent $P$ trusts agent $Q$ whereas agent $Q$ might not trust agent $P$ [1]. This model (1) is more suitable for the latter case. This is the limitation of this model. However, if one agrees that trust is temporary, whereas distrust or mistrust is ubiquitous, then this model is still of practical significance.

3. Transitivity. If agent $P$ trust agent $Q$, and agent $Q$ trust agent $R$, then agent $P$ trust agent $R$. For example, it is very common in e-commerce if customer $A$ trusts his friend $B$, and $B$ trusts eBay.com, then $A$ trusts eBay.com. This is a kind of transitive trust or trust propagation in customer-to-business e-commerce. However, trust is not transitive in some cases. For instance, customer $A$ trusts his friend $B$, and $B$ trusts an e-commerce website, however, $A$ does not trust this website.

Therefore, a trust relation is conditionally symmetric and transitive [22].

In reality, the condition (1) can be weakened to three different possibilities that lead to "agent $P$ trusts agent $Q$."

$$
\begin{array}{ll}
\text { 1. } & K_{P} \subseteq K_{Q} \\
\text { 2. } & R_{P} \subseteq R_{Q} \\
\text { 3. } & K_{P} \subseteq K_{Q} \text { and } R_{P} \subseteq R_{Q}
\end{array}
$$


The first possibility is that "agent $P$ trusts agent $Q$ " because agent $Q$ has more knowledge and experience than agent $P$. For example, in a primary school, a student trusts his teacher, because the latter has more knowledge and experience than he. Therefore, the trust resulting from this possibility is called knowledge-based trust. In other words, knowledge-based trust is based on one's knowledge and experience about competencies, motives, and goals of the agent $[1,16]$.

The second possibility is that "agent $P$ trusts agent $Q$ " because agent $Q$ has more reasoning methods or problem solving methods than agent $P$. For example, in a system development team, a young team member trusts his team leader, because the latter has more problem solving methods than the former in systems analysis. Therefore, the trust resulting from the second possibility is called reasoning-based trust. This implies that this trust is based on one's reasoning and problem solving abilities $[1,22]$

The third possibility is that "agent $P$ trusts agent $Q$ " because agent $Q$ has more knowledge, experience and more reasoning methods or problem solving methods than agent $P$. For example, a patient trusts an experienced doctor working in a clinic, because the doctor has more knowledge, experience and more methods in diagnosis and treatment. Therefore, the trust resulting from the third possibility is called hybrid trust. In other words, hybrid trust is a combination of knowledge-based trust and reasoning-based trust [16].

It is very common for agents $P$ and $Q$ in e-commerce or MECS to have a hybrid trust relationship, because the knowledge and reasoning methods of an agent are usually considered as his ability or reputation in particular in the case of trust as discussed in others' work such as Xiu and Liu [22]. Based on this idea, this investigation is more fundamental than reputation-based trust [6] and ability-based trust (see later).

\section{Experience-Based Trust in E-Commerce}

Experience-based trust has also been drawn attention in e-commerce [8]. Experiencebased trust is to use past experiences to help build the trust in the potential buyers or sellers in e-commerce. The feedback from other peers to the potential buyers or sellers also plays an important role in building of trust which is the basis for recommendation and judgement on the potential buyers or sellers [23]. This section extends reasoning-based trust and hybrid trust to experience-based trust in ecommerce.

From a logic viewpoint, there are eight basic inference rules for performing EBR [15], which are summarized in Table 1, and constitute the fundamentals for all EBR paradigms [13]. The eight inference rules are listed in the first row, and their corresponding general forms are shown in the second row respectively. The eight inference rules are modus ponens (MP), modus tollens (MT), abduction, modus ponens with trick (MPT), modus tollens with trick (MTT), abduction with trick (AT), inverse modus ponens (IMP), inverse modus ponens with trick (IMPT). For simplicity, we denote them as $R=\left\{R_{1}, R_{2}, R_{3}, R_{4}, R_{5}, R_{6}, R_{7}, R_{8}\right\}$. The first four of them have been used in computer science, mathematics, mathematical logic, and 
other sciences $[13,14]$. The rest were proposed in the past few years [13, 15], to our knowledge. However, they are all the abstraction and summary of EBR in real world problems.

Table 1. Experience-based reasoning: Eight inference rules

\begin{tabular}{|c|c|c|c|c|c|c|c|}
\hline MP & MT & $\begin{array}{c}\text { abdu- } \\
\text { ction }\end{array}$ & MTT & AT & MPT & IMP & IMPT \\
\hline$P$ & $\neg Q$ & $Q$ & $\neg Q$ & $Q$ & $P$ & $\neg P$ & $\neg P$ \\
$\frac{P \rightarrow Q}{\therefore Q}$ & $\frac{P \rightarrow Q}{\therefore \neg P}$ & $\frac{P \rightarrow Q}{\therefore P}$ & $\frac{P \rightarrow Q}{\therefore P}$ & $\frac{P \rightarrow Q}{\therefore \neg P}$ & $\frac{P \rightarrow Q}{\therefore \neg Q}$ & $\frac{P \rightarrow Q}{\therefore \neg Q}$ & $\frac{P \rightarrow Q}{\therefore Q}$ \\
\hline
\end{tabular}

It should be noted that "with trick" is only an explanation for such models. One can give other semantic explanations for them. For example, one can use exception or deception to explain them in the context of the stock market or systems diagnosis [13].

In the rest of this section, we will discuss reasoning-based trust in the context of EBR.

As is well known, one reasoning or problem solving consists of a chain of inference rules. In other words, one agent $P$ 's reasoning method consists of a few fundamental inference rules. Therefore, we can use inference rules (how many times has an inference rule been used? how many inference rules an agent has used for a problem solving?) to measure the reasoning methods or problem solving methods. Based on this discussion, let $R=\left\{R_{1}, R_{2}, R_{3}, R_{4}, R_{5}, R_{6}, R_{7}, R_{8}\right\}, R_{P} \subseteq R$ and $R_{Q} \subseteq R$, then agent $P$ trusts agent $Q$ with respect to EBR iff $R_{P} \subseteq R_{Q}$. This implies that "agent $P$ trusts agent $Q$ " because in the problem solving agent $Q$ has used more inference rules in particular, more problem solving methods in general of EBR than agent $P$. For example, in a transaction of e-commerce, agent $P$ trusts agent $Q$ with respect to EBR, because agent $Q$ uses modus ponens (MP), modus tollens (MT), abduction, inverse modus ponens (IMP) on some e-commerce occasions; that is, $R_{Q}=\left\{R_{1}, R_{2}, R_{3}, R_{7}\right\}$ and agent $P$ only uses inference rules, $R_{P}=\left\{R_{1}, R_{2}, R_{3}\right\}$. In particular, in e-commerce negotiation, a seller agent might use any possible inference rules of the EBR to make an e-customer agent trust him [14]. Normally, an e-customer uses less inference rules of EBR in e-commerce negotiation.

\section{Measure and Evaluation of Trust}

Tweedale and Cutler examine trust in multiagent systems and note the measure of trust [18]. However, they have not gone into it. In what follows, we will introduce a unified measure of trust based on the discussion of the previous section.

Generally, let the cardinality (size) of knowledge set $K$ and reasoning methods set $R$ be $|K|$ and $|R|$ respectively. Then the trust degree of agent $P$ in agent $Q$ can be denoted as 


$$
T(P, Q)=\alpha\left(\frac{1-\left|K_{P}\right|}{\left|K_{Q}\right|}\right)+(1-\alpha)\left(\frac{1-\left|R_{P}\right|}{\left|R_{Q}\right|}\right)
$$

where when $\alpha=1, T(P, Q)$ is the knowledge-based trust degree of agent $P$ in agent $Q$. When $\alpha=0, T(P, Q)$ is the reasoning-based trust degree of agent $P$ in agent $Q$. When $0<\alpha<1, T(P, Q)$ is the hybrid trust degree of agent $P$ in agent $Q$. For example, if knowledge-based trust degree of agent $P$ in agent $Q$ is 0.8 , the reasoning-based trust degree of agent $P$ in agent $Q$ is 0.4 , and $\alpha=0.7$, then hybrid trust degree of agent $P$ in agent $Q$ is $T(P, Q)=0.7 \times 0.8+0.3 \times 0.4=0.68$.

Further, $\left(1-\left|K_{P}\right|\right) / K_{Q}$ implies that agent $P$ 's trust degree is greater whenever the size of knowledge set of the agent $Q$ is greater than that of agent $P$ taking into account (1). Similarly, $\left(1-\left|R_{P}\right|\right) / R_{Q}$ implies that agent $P$ 's trust degree is greater whenever the size of reasoning methods of the agent $Q$ is greater than that of agent $P$. The key idea behind it is that agent $P$ easily trust agent $Q$ if the latter has more knowledge and experience or problem solving ability than agent $P$ taking into account (1). This case usually happens when a student trusts his teacher. With the age increasing the trust between any two persons will be decreasing. In other words, it is more difficult for one to trust others in the adult world. Therefore, $\left(1-\left|K_{P}\right|\right) / K_{Q}$ or $\left(1-\left|R_{P}\right|\right) / R_{Q}$ will be decreasing when the size of knowledge set of the agent $P$ approaches to that of agent $Q$ or the size of reasoning methods of the agent $P$ approaches to that of agent $Q$. For simplicity, we use $T(P, Q)$ to denote either knowledge based trust degree or reasoning trust degree or hybrid trust degree and do not differ one from another without specification.

\section{A Case-Based Model for Ability Based Trust}

The intelligent model for trust introduced in Section 3 is not valid for dealing with the ability-based trust. This section will focus on this kind of trust.

Ability-based trust is related to the trustee's knowledge, skills or competency to perform as expected [22]. This kind of trust is related to one agent $P$ and other agents or in particular agent team $Q=\left\{Q_{1}, Q_{2}, \ldots, Q_{n}\right\}$ with respect to task $t$. Assume that for the agent $P$, the satisfaction value that agent $Q_{i}, i \in\{1,2, \ldots, n\}$ receives for completing task $t$ is $s\left(P, Q_{i}, t\right)$, where $s(,, \quad) \in[0,1]$, then the trust degree of agent $P$ in agent $Q_{i}$ can be considered as $s\left(P, Q_{i}, t\right)$ [23] (more generally, agent $P$ should be replaced by a special standard for completing a task.); that is,

$$
T\left(P, Q_{i}, t\right)=s\left(P, Q_{i}, t\right)
$$

Therefore, the most trustworthy agent of agent $P$ with respect to task $t$ should be agent $Q_{k}$ with a trust degree $T\left(P, Q_{k}, t\right)$ and $k \in\{1,2, \ldots, n\}$ such that for any $i \in\{1,2, \ldots, n\}, T\left(P, Q_{k}, t\right) \geq T\left(P, Q_{i}, t\right)$. We can briefly denote this as

$$
T(P, Q, t)_{\max }=T\left(P, Q_{k}, t\right)
$$

Now there is a new task $t_{0}$ to be done by one of the agents in $Q . t_{0}$ is similar to task $t$. The question is who should be most trustworthy agent for agent $P$ to complete this task. In what follows we answer this question. 
Let $t$ be similar to $t_{0}$, then $T\left(P, Q_{i}, t\right) \approx T\left(P, Q_{i}, t_{0}\right), \approx$ is a similarity metric [14], then the most trustworthy agent of agent $P$ with respect to task $t$, agent $Q_{k}$ with a trust degree $T\left(P, Q_{k}, t\right)$ that satisfies (4) will be recommended based on case-based reasoning [14] to complete the new task $t_{0}$, which is a kind of experience-based recommendation.

\section{Concluding Remarks}

This paper examined experience-based trust, knowledge-based trust, inferencebased trust and case-based trust in e-commerce and their interrelationships from an intelligent systems viewpoint. The proposed approach will improve a formal understanding of trust and facilitate research and development of trust, multiagent systems, e-commerce and e-services.

In future work, we will develop a system prototype for the multiagent case based trust management system, which can be used for business recommendation and negotiation. We will also examine scalable trust in e-commerce and e-services.

\section{References}

M. Branchaud and S. Flinn, 2004, xTrust: A scalable trust management infrastructure, In Proc 2nd Annual Conf on Privacy, Security and Trust, Fredericton, New Brunswick, Canada, 14-15 October, pp. 207-218.

C. Castelfranchi, and Y.H. Tan (eds), 2001, Trust and Deception in Virtual Societies, Kluwer Academic Publishers, Norwell MA, USA.

G. Chen, Z. Li, Z. Cheng, Z. Zhao, and H. Yan, 2005, A fuzzy trust model for multiagent systems, LNCS 3612, Springer, Berlin, pp. 444-448.

G Finnie, Sun Z, and Barker J, 2005, Trust and deception in multi-agent trading systems: A logical viewpoint, In: Proc. 11th Americas Information Systems (AMCIS2005), The Association for Information Systems, Aug 11-14, 2005 Omaha, NE, USA, pp. 1020-1026.

D. H. McKnight, V. Choudhury, and C. Kacmar, 2002, The impact of initial customer trust on intentions to transact with a website: A trust building model, J of Strategic Information Systems, 11, 297-323.

M. Nielsen and K. Krukow, 2004, On the formal modelling of trust in reputation-based systems, LNCS 3113, Berlin Heidelberg Springer-Verlag, pp. 192-204.

N.J. Nilsson, 1998, Artificial Intelligence: A New Synthesis. San Francisco: Morgan Kaufmann Publishers.

O. Olsson, 2002, Trust in eCommerce - the ontological status of trust, Proc ECOM-02 Electronic Commerce - Theory and Applications, B. Wiszniewski (ed), Gdansk, pp. 89-96.

P. A. Pavlou, 2003, Customer acceptance of electronic commerce: Integrating trust and risk with the technology acceptance model, Intl $\mathrm{J}$ of Electronic Commerce 7(3), 135-161.

Salam AF, lyer L, Palvia P, and Singh R, 2005, Trust in e-commerce, Comm of The ACM, 48(2), 73-77.

S. Schmidt, R. Steele, T. Dillion and E. Chang, 2005, Applying a fuzzy trust model to ecommerce systems, LNAI 3809, Springer,pp 318-329. 
C.V. Slyke, F. Belanger, and C.L. Comunale, 2004, Factors influencing the adoption of webbased shopping: The impact of trust, The Data Base for Advances in Information Systems, vol. 35 , no. 2 .

Z. Sun and G. Finnie, 2004a, Experience based reasoning for recognizing fraud and deception. In: Proc. Intl Conf on Hybrid Intelligent Systems (HIS 2004), December 6-8, Kitakyushu, Japan, IEEE Press, pp. 80-85.

Z. Sun and G. Finnie, 2004, Intelligent Techniques in E-Commerce: A Case-based Reasoning Perspective. Berlin Heidelberg: Springer-Verlag.

Z. Sun and G. Finnie, 2007, A fuzzy logic approach to experience based reasoning, Intl J Intell Syst. 22(8) 867-889.

Z. Sun, Y. Li, and S. Zhao, 2007, Trust, deception and security in e-commerce. In Columbus F (ed) E-Commerce Research and Trends, NY: Nova Science Publishers. In press.

Y.H. Tan and W. Thoen, 2001, Toward a generic model of trust for electronic commerce. Intl $\mathrm{J}$ of Electronic Commerce 5(2), 61-74.

J.Tweedale and P. Cutler, 2006, Trust in multiagent systems, LNCS 4252, Berlin Heidelberg Springer-Verlag, pp. 479-485.

Uslaner EM, 2004, Trust online, trust offline, Comm of The ACM, 47(4), 28-29.

T. Verhagen, S. Meents, and Y. H. Tan, 2006, Perceived risk and trust associated with purchasing at electronic marketplaces, European J of Information Systems, 15, 542-555.

S. C. Wingreen, S.L. Baglione, 2005, Untangling the antecedents and covariates of ecommerce trust: institutional trust vs. knowledge-based trust, Electronic Markets, 15 (3), 246 260.

D. Xiu, and Z. Liu, 2005, A formal definition for trust in distributed systems, LNCS 3650 , Springer Verlag, Berlin Heidelberg, pp. 482-489.

L. Xiong and L. Liu, 2002, Building trust in decentralized peer-to-peer electronic communities, In Proc Intl Conf on Electronic Commerce Research (ICECR-5), Montreal, Canada, October, 2002 